\title{
STRESS AND COPING AMONG MEDICAL OFFICERS IN KERALA: A CROSS-SECTIONAL STUDY
}

\author{
Anvar Sadath ${ }^{*}$, Shibu Kumar ${ }^{2}$ \\ ${ }^{1}$ Psychiatric Social Worker, Dept. of Psychiatry, Government Medical College, Kollam. \\ ${ }^{2}$ Assistant Professor in Psychiatry, Institute of Mental Health and Neurosciences, Kozhikode. \\ *Correspondence: Dept. of Psychiatry, Government Medical College, Kollam. E-mail: anvarvakkayil@gmail.com
}

\begin{abstract}
Background: There is strong evidence to suggest that medical practitioners experience high levels of stress and it affects their work performance and quality of medical care. Adaptive and maladaptive coping strategies can influence the extent and severity of stress. However, empirical studies examining stress and coping among medical practitioners are less from Kerala.

Methods: Using a cross-sectional research design, we examined stress and coping among 31 medical officers in Kerala. The participants were recruited during the annual meeting of Kerala Government Medical Officers Association (KGMOA) held at Nilambur. Perceived Stress Scale and Brief Cope Inventory were used to measure their stress and coping. Descriptive and bivariate analysis were used to interpret the data.

Results: Participants experienced moderate level of stress. The stress was significantly correlated with denial $(\mathrm{r}=.646)$, substance use $(\mathrm{r}=.667)$, use of emotional support $(\mathrm{r}=-.674)$ use of instrumental support $(\mathrm{r}=-.612)$ positive reframing $(\mathrm{r}=-.510)$ and planning $(\mathrm{r}=-.459)$.

Conclusion: Future studies may examine the predictive validity of the identified coping variables in a large sample. It is highly recommended that stress management and coping skill training programmes be organized for the medical officers in Kerala.
\end{abstract}

Keywords: stress, coping, medical officers

\section{BACKGROUND}

Occupational status, in varying degree, has been associated with stress. ${ }^{1}$ Researchers have consistently identified six main factors which are sources of occupational stress common to all jobs. It includes factors intrinsic to the job, career development, role-based stress, relationships at work, organisational structure, and work/home interface. ${ }^{1}$ Although different occupational groups describe their work as stressful, it seems that some occupations carry more stress than others. Medicine is certainly a stressful profession with prolonged working hours, ethical dilemmas, and difficult patients. $^{2}$ There is strong evidence to show that medical practitioners experience higher stress, 3,4 which is a strong determining factor for poor work performance and quality of medical care. ${ }^{5}$ The medical practitioners are in continuous contact with disease, sufferings, distress, death and dying which may contribute to stress. $^{6}$ The intensity of workload, coping with diagnostic uncertainty, working alone, working unsocial hours, ${ }^{7}$ fears of making mistakes that can lead to serious consequences, working with uncooperative colleagues, doing work that is mentally straining, and feeling of being underpaid may also increase the 
stress. ${ }^{8}$ Stress has many adverse effects on medical practice, including prescribing errors, lack of teamwork, more patients' complaint, and sickness absence. ${ }^{9}$ The higher stress among these doctors can lead to alcohol and drug abuse, interpersonal relationship difficulties, depression and anxiety, and even suicide. ${ }^{9}$ The work performance and quality of medical care administered to the patients can be undermined by overstressed physicians.

Coping is a process of managing demands (external or internal) that are appraised as taxing or exceeding the resources of the person. ${ }^{10}$ In general, coping can be problem focussed or emotion focussed. The former is aimed at problem-solving or doing something to alter the sources of the stress, while the latter is aimed at reducing or managing the emotional distress associated with the situation. Even though most stressors elicit both types of coping, problem-focused coping tends to predominate when people feel that something constructive can be done, while emotion-focused coping tends to predominate when people feel that the stressor is something that must be endured. ${ }^{11}$ The type of coping can increase or diminish the effects of stress, and different types of coping strategies can have protective or harmful effects on individuals' health and wellbeing. ${ }^{12}$ The use of social support, planning, acceptance, etc. are expected to protect from the effects of stress, while substance use and denial can increase stress. ${ }^{12,13}$

Stress and coping are widely researched psychological constructs, yet there is a paucity of research on stress among the doctors in Kerala. Since cultural factors mediate stress, ${ }^{14}$ the studies on stress from other countries or even from other states may have less application in the Kerala context. Given this background, we conducted the study with the following objectives:

1. To study the extent of stress among medical officers in Kerala

2. To study the relationship between stress and coping variables

\section{METHODS}

The sample for this cross-sectional study constituted 31 voluntarily participated Government Medical Officers in Malappuram District. All the participants were registered members of Kerala Government Medical Officers Association (KGMOA), Malappuram Chapter. Using consecutive sampling technique, the participants were recruited during KGMOA's annual family meet called 'Aaranyakam' held in Teak Town Resort, Mampad, Nilambur, Kerala on $12^{\text {th }}$ February 2017. Among the 225 medical officers registered, 31 members participated in the study. Majority of the registered members could not participate as they were not available at the time of data collection or they were busy in organizing the program. The study was conducted as a part of a stress management programme for these doctors.

We used a self-prepared demographic data sheet to collect the personal and professional details of the participants. Along with it, the following standardised instruments were used to measure their perceived stress and coping. We used the original English versions of the scales as all the participants were able to understand them. Informed consent in writing was obtained from each participant prior to their participation in this study.

Perceived Stress Scale (PSS): PSS is a widely used brief psychological instrument for measuring the perception of stress. ${ }^{15}$ It has ten items that measure the degree to which the situations in one's life are appraised as stressful. Items were designed to tap how unpredictable, uncontrollable, and overloaded respondents find their lives. The items are easy to understand, and the response alternatives are simple to grasp. Moreover, the questions are of a general nature and hence they are relatively free of content specific to any subpopulations. The questions in the scale ask about feelings and thoughts during the last month. The scoring is done on a four-point scale ranging from 0 to 4 ( $0=$ Never, $1=$ Almost Never, $2=$ Sometimes, $3=$ Fairly Often, $4=$ Very Often). The aggregated score of $0-13$ is considered low stress, 14-26 is moderate stress, and scores ranging from 27-40 are considered high perceived stress. 
The coefficient alpha reliability ranges from .84 to .86 , and the test-retest reliability of the scale is $.85 .^{15}$ This instrument has been validated and used in many Indian ${ }^{16,17}$ and Asian studies. ${ }^{18,19}$

Brief COPE: The Brief COPE is the abbreviated version of the COPE Inventory and assesses dispositional as well as situational coping efforts. ${ }^{13}$ The 28-item Brief COPE consists of 14 subscales (see Table 2) and it has been used extensively to examine the relationship between various coping strategies and psychological outcomes. It is a fourpoint Likert scale ( $I=$ "I haven't been doing this at all"; 2 = "I've been doing this a little bit"; $3=$ "I've been doing this a medium amount"; $4=$ "I've been doing this a lot"). The Brief COPE was chosen in preference to other coping questionnaires because it encompasses a broad range of coping strategies and it can be presented in a situational rather than a trait format. Thus, this instrument is helpful to measure the medical practitioners' coping specifically arising from their situational demands.

\section{DATA ANALYSIS}

Data in this study were analysed using SPSS 13 version. Descriptive analysis was performed by using frequencies, percentage, mean and standard deviation. Bivariate analysis was performed by using Pearson's Correlation.

\section{RESULTS}

Majority of the medical officers were male (54.8\%), while all of them were married. Highest professional qualification of most of them was MBBS (77.4\%). Majority of the respondents' spouses were also medical practitioners. Most of them were working in general cadre $(64.5 \%)$. The mean age of the medical officers was 37.41 years and they had an average 10.29 years of experience in their field (see Table 1).

The average stress score was $23.45 \pm 6.29$, which indicated moderate perceived stress.
Table 1: Socio-demographic characteristics of the study participants ( $n=31$ )

\begin{tabular}{|l|l|}
\hline Variables & $\mathbf{n}(\%)$ \\
\hline Gender & $17(54.8)$ \\
\hline Male & $14(45.2)$ \\
\hline Female & \\
\hline Marital status & $31(100)$ \\
\hline Married & - \\
\hline Unmarried & $24(77.4)$ \\
\hline Professional qualification & \\
\hline MBBS only & $07(22.6)$ \\
\hline MBBS\&MD & \\
\hline Spouse job & $20(64.5)$ \\
\hline Medical practitioner & $11(35.5)$ \\
\hline Other profession & \\
\hline Cadre & $20(64.5)$ \\
\hline General & $08(25.8)$ \\
\hline Speciality & $03(9.7)$ \\
\hline Administration & Mean (SD) \\
\hline & $37.41(6.90)$ \\
\hline Age & $10.29(6.26)$ \\
\hline Years of experience &
\end{tabular}

Table 2: Correlation of perceived stress with various domains of coping

\begin{tabular}{|c|c|}
\hline Coping domains & $\mathbf{r}$ \\
\hline Self-distraction & -.255 \\
\hline Active coping & -.303 \\
\hline Denial & $.646^{* *}$ \\
\hline Substance use & $.667^{* *}$ \\
\hline Use of emotional support & $-.674^{* *}$ \\
\hline Use of instrumental support & $-.612^{* *}$ \\
\hline Behavioral disengagement & .047 \\
\hline Venting & -.031 \\
\hline Positive reframing & $-.510^{* *}$ \\
\hline Planning & $-.459^{* *}$ \\
\hline Humor & -.152 \\
\hline Acceptance & $-.401^{*}$ \\
\hline Religion & .010 \\
\hline Self-blame & $.556^{* *}$ \\
\hline
\end{tabular}


Table 2 shows the relationship of perceived stress with various domains of coping in medical officers. As the table shows, perceived stress was correlated with respondents' denial and substance use coping. It indicates that higher stress they experience possibly increased their denial and substance use behaviour or vice versa. The respondents' use of emotional support and instrumental support was negatively correlated with their perceived stress. Higher the use of emotional and instrumental support, lesser was their experience of stress. Similarly, positive reframing, planning, and acceptance coping were negatively correlated with stress, which indicated less stress experiences for those who use them. Finally, self-blame was correlated with stress. It indicates that high selfblame coping possibly increases stress or vice versa.

\section{DISCUSSION}

Stress among medical practitioners has always been a topical issue because their service involves taking care of other peoples' lives and even minor mistakes or errors could be costly and sometimes irreversible. ${ }^{20}$ It is thus expected that the medical doctor himself must be in a perfect state of mind devoid of morbid worries and anxieties. ${ }^{20} \mathrm{We}$ found a moderate level of perceived stress among the Government medical officers in Kerala. This finding is slightly different from a study among the doctors working in critical care units of a private hospital in India which reported a moderate to high stress among the doctors. ${ }^{21}$ Another study among 930 resident doctors from four medical colleges at Delhi had found severe stress in a majority of the residents. Important reasons of stress as perceived by them included long duty hours, departmental academic activities, financial constraints, and family problems. ${ }^{22}$ However, it has to be noted that our study is among the medical officers working in the government sector and not with the doctors in private hospitals or residents and thus expected to have some variations in stress experience. Possibly, the stress variations could be due to the difference in job security, work environment, the prospects for career advancement, workload, and the type of patients they manage. An earlier study reported high job satisfaction among the doctors in government medical colleges. ${ }^{23}$ Moreover, the healthcare system in Kerala is advanced with better infrastructure and staff, ${ }^{24}$ which may also contribute to the reduction of stress.

Evidently, the bivariate analysis revealed associations between stress and coping. The stress was high with denial and substance use while it was lesser with social support, positive reframing and acceptance or vice versa. Although not in this population, the association of stress and denial, ${ }^{25}$ and stress and substance use behaviours ${ }^{26}$ are well established. A recent study from Pakistan among resident doctors found adaptive stress-coping strategy highest with religion, followed by planning, acceptance and active coping, while the maladaptive stress-coping strategy was higher with self-blame, followed by self-distraction and venting. ${ }^{27}$ Nevertheless, we could not replicate most of these findings in the current study. Others reported an inverse correlation between stress and problem-focused coping and positive correlation between stress and emotion-focused and avoidance coping. ${ }^{28}$ Many other individual and structural factors are associated with stress which we didn't examine in this study. For example, the number of working hours, dealing with difficult patients, workloads, ${ }^{29}$ level of responsibilities, ${ }^{21}$ job satisfaction, having close friends, spending time with family/friends, ${ }^{22}$ and gender status ${ }^{30}$ are all potential variables mediating stress.

The strength of the study includes the fact that it is a maiden attempt to understand the stress and coping among the medical officers in Kerala. However, the results need to be interpreted with the consideration of small sample size, cross-sectional nature, and the fact that the participants were government medical officers who were part of one association. Moreover, the data for the study was collected during their association meeting and family get-together, which could have mediated their stress experience. Future studies may examine the predictive validity of the identified coping variables in a large representative sample. Prospective studies also can be planned to overcome the limitation of the cross-sectional data and it will help us to understand the stability of stress and 
coping over a period. Although we did not examine the substance use behaviour in detail, stress was found to be associated with substance use. Thus, we recommend future studies to address this issue in detail.

\section{CONCLUSION}

We found a moderate level of stress among the Government Medical officers in Kerala. The stress was related to many coping variables including denial, substance use, social support, positive reframing, and planning. We strongly recommend stress management and coping skill training programmes for the medical officers in Kerala.

\section{REFERNCES}

1. Cooper CL, Marshall J. Occupational sources of stress: A review of the literature relating to coronary heart disease and mental ill health. From Stress to Wellbeing Volume 1: Springer; 2013: 3-23.

2. Hassan M, Hussain T, Ahmed SM, Fraz TR, Rehmat Z. Perceived stress and stressors among house officers. Indian J Occup Environ Med 2014; 18(3): 145-9.

3. Association BM. Stress and the medical profession: British Medical Association London; 1992.

4. Rout UR, Rout JK. Stress management for primary health care professionals: Springer Science \& Business Media; 2007.

5. Makames RA, Alkoot EM, Al-Mazidi BM, El-Shazly MK, Kamel MI. Sources and expressions of stress among physicians in a general hospital. Alexandria Journal of Medicine 2012; 48(4): 361-6.

6. Bates E. Doctors and their spouses speak: stress in medical practice. Sociology of health \& illness 1982; 4(1): 25-39.

7. Williams S, Dale J, Glucksman E, Wellesley A. Senior house officers' work related stressors, psychological distress, and confidence in performing clinical tasks in accident and emergency: a questionnaire study. BMJ 1997; 314(7082): 713.

8. Yusoff M, Jie T, Esa A. Stress, stressors and coping strategies among house officers in a Malaysian hospital. ASEAN Journal of Psychiatry 2011; 12(1): 85-94.

9. Shapiro SL, Shapiro DE, Schwartz GE. Stress management in medical education: a review of the literature. Acad Med 2000; 75(7): 748-59.

10. Folkman S. Stress: appraisal and coping. Encyclopedia of behavioral medicine: Springer; 2013: 1913-5.

11. Folkman S, Lazarus RS. An analysis of coping in a middleaged community sample. J Health Soc Behav 1980; 219-39.

12. Lazarus RS. Coping theory and research: past, present, and future. Psychosom Med 1993; 55(3): 234-47.

13. Carver CS. You want to measure coping but your protocol'too long: Consider the brief cope. Int J Behav Med 1997; 4(1): 92100.

14. Post DM, Weddington $\mathrm{W}$. The impact of culture on physician stress and coping. J Natl Med Assoc 1997; 89(9): 585-90.
15. Cohen S, Kamarck T, Mermelstein R. A global measure of perceived stress. J Health Soc Behav 1983: 385-96.

16. Chakraborti A, Ray P, Sanyal D, et al. Assessing perceived stress in medical personnel: in search of an appropriate scale for the Bengali population. Indian J Psychol Med 2013; 35(1): 29.

17. Sadath A, Muralidhar D, Varambally S, Gangadhar B, Jose JP. Do stress and support matter for caring? The role of perceived stress and social support on expressed emotion of carers of persons with first episode psychosis. Asian J Psychiatr 2017; 25: 163-8.

18. Al-Dubai SA, Barua A, Ganasegeran K, Jadoo SA, Rampal KG. Concurrent validity of the Malay version of Perceived Stress Scale (PSS-10). Asian J Psychiatr 2014; 15(1): 8-13.

19. Leung DY, Lam T-h, Chan SS. Three versions of Perceived Stress Scale: validation in a sample of Chinese cardiac patients who smoke. BMC public health 2010; 10(1): 513.

20. Familoni OB. An overview of stress in medical practice. Afr Health Sci 2008; 8(1): 6-7.

21. Amte R, Munta K, Gopal P. Stress levels of critical care doctors in India: A national survey. Indian J Crit Care Med 2015; 19(5): 257-64.

22. Saini N, Agrawal S, Bhasin S, Bhatia M, Sharma A. Prevalence of stress among resident doctors working in Medical Colleges of Delhi. Indian J Public Health 2010; 54(4): 219-23.

23. Bhattacherjee S, Ray K, Kumar Roy J, Mukherjee A, Roy H, Datta S. Job Satisfaction among doctors of a government medical college and hospital of Eastern India. Nepal J Epidemiol. 2016; 6(3): 596-602.

24. Nabae K. The health care system in Kerala: its past accomplishments and new challenges. Journal of the National Institute of Public Health 2003; 52(2): 140-5.

25. Houston BK. Viability of coping strategies, denial, and response to stress. J Pers 1973; 41(1): 50-8.

26. Sinha R. Chronic Stress, Drug Use, and Vulnerability to Addiction. Ann N Y Acad Sci 2008; 1141: 105-30.

27. Alosaimi FD, Almufleh A, Kazim S, Aladwani B. Stresscoping strategies among medical residents in Saudi Arabia: A cross-sectional national study. Pak J Med Sci 2015; 31(3): 5049.

28. Vinothkumar M, Arathi A, Joseph M, Nayana P, Jishma E, Sahana U. Coping, perceived stress, and job satisfaction among medical interns: The mediating effect of mindfulness. Ind Psychiatry J 2016; 25(2): 195-201.

29. Boran A, Shawaheen M, Khader Y, Amarin Z, Hill Rice V. Work-related stress among health professionals in northern Jordan. Occup Med (Lond) 2012; 62(2): 145-7.

30. Rashid I, Talib P. Occupational Stress and Coping Styles among Doctors: Role of Demographic and Environment Variables. Vision 2015; 19(3): 263-75.

Source of support: None

Conflict of interest: None declared

First submitted: $21^{\text {st }}$ November 2017

Published online: $30^{\text {th }}$ March 2018 